\title{
Diversity and Distribution of liverworts across habitats and altitudinal gradient at Pachmarhi Biosphere Reserve (India)
}

\author{
Reesa Gupta and Ashish Kumar Asthana \\ Bryology Laboratory, CSIR- National Botanical Research Institute, Lucknow - 226001, India
}

\section{Article history}

Received: 26 August 2016

Accepted: 02 September 2016

Published: 01 October 2016

(c) Gupta and Asthana (2016)

Editor

K. K. Sabu

Publisher

Horizon e-Publishing Group

Corresponding Author

A.K. Asthana

$\square_{\text {drakasthanaster@gmail.com }}$

\begin{abstract}
The present study elucidates the distribution of liverworts (Marchantiophyta) in various habitats and across the altitudinal gradients at Pachmarhi Biosphere Reserve (PBR), central India. The liverwort diversity was assessed in selected habitats at each site viz. soil, wet rocks, dry rocks, soil covered rocks, stony walls (terricolous habitats) and epiphytic habitat. Three altitudinal gradients were considered for distributional assessment. In all, 41 liverworts belonging to 21 genera and 15 families were encountered. Among the three altitudinal zones, 17 taxa were found at lower altitudinal gradient $(400-800 \mathrm{~m})$ whereas 12 liverworts were found at the higher altitudinal gradient (1001-1400 m). Maximum taxa (33) were present at the middle altitudinal zone $(801-1000 \mathrm{~m})$. The sites at middle altitudes furnished amicable conditions for the growth of bryophytes. In general, rocks, both moist and dry formed the most pertinent habitat for the liverworts. Evidently, the middle altitudinal gradient emerged as the altitudinal range harbouring maximum liverworts.
\end{abstract}

\section{Keywords}

Bryophytes; liverworts; habitats; altitudinal gradients

Gupta, R. and A. K. Asthana. 2016. Diversity and Distribution of liverworts across habitats and altitudinal gradient at Pachmarhi Biosphere Reserve (India). Plant Science Today 3(4): 354-359. http://dx.doi.org/10.14719/pst.2016.3.4.263

\section{Introduction}

Pachmarhi Biosphere Reserve (PBR) lies in the Hoshangabad, Betul and Chhindwara Districts of Madhya Pradesh and was designated under MAB Programme in 1999. The present study elucidates the distribution of liverworts (Marchantiophyta) at various habitats across the altitudinal gradients in this Biosphere Reserve. This region is considered as the richest region of central India in terms of cryptogam diversity. PBR encompasses three conservation units viz. the Pachmarhi Sanctuary, Satpura National Park and Bori Sanctuary along with the areas surrounding these. As far as the cryptogamic flora of PBR is concerned, researchers have ventured into the studies of algae, lichens, bryophytes and pteriodophytes from later part of the $20^{\text {th }}$ century. The studies on bryophytes at PBR were initiated way back in 1950s by Pande and Srivastava (1952) probably due to the substantial number of bryophytes anticipated from the region. Further workers listed few species of bryophytes, mainly the liverworts from the area, viz. Lal and Parihar (1979), Jain and Kaul (1985), Patidar et al (1985) and Kaul et al. (1995, 1995a). In the past decades listings of bryoflora of Pachmarhi and neighbouring areas have been provided by Kaul (2001), Singh and Kaul (2002) and most recently by Sharma and Alam (2011). The previous efforts on 


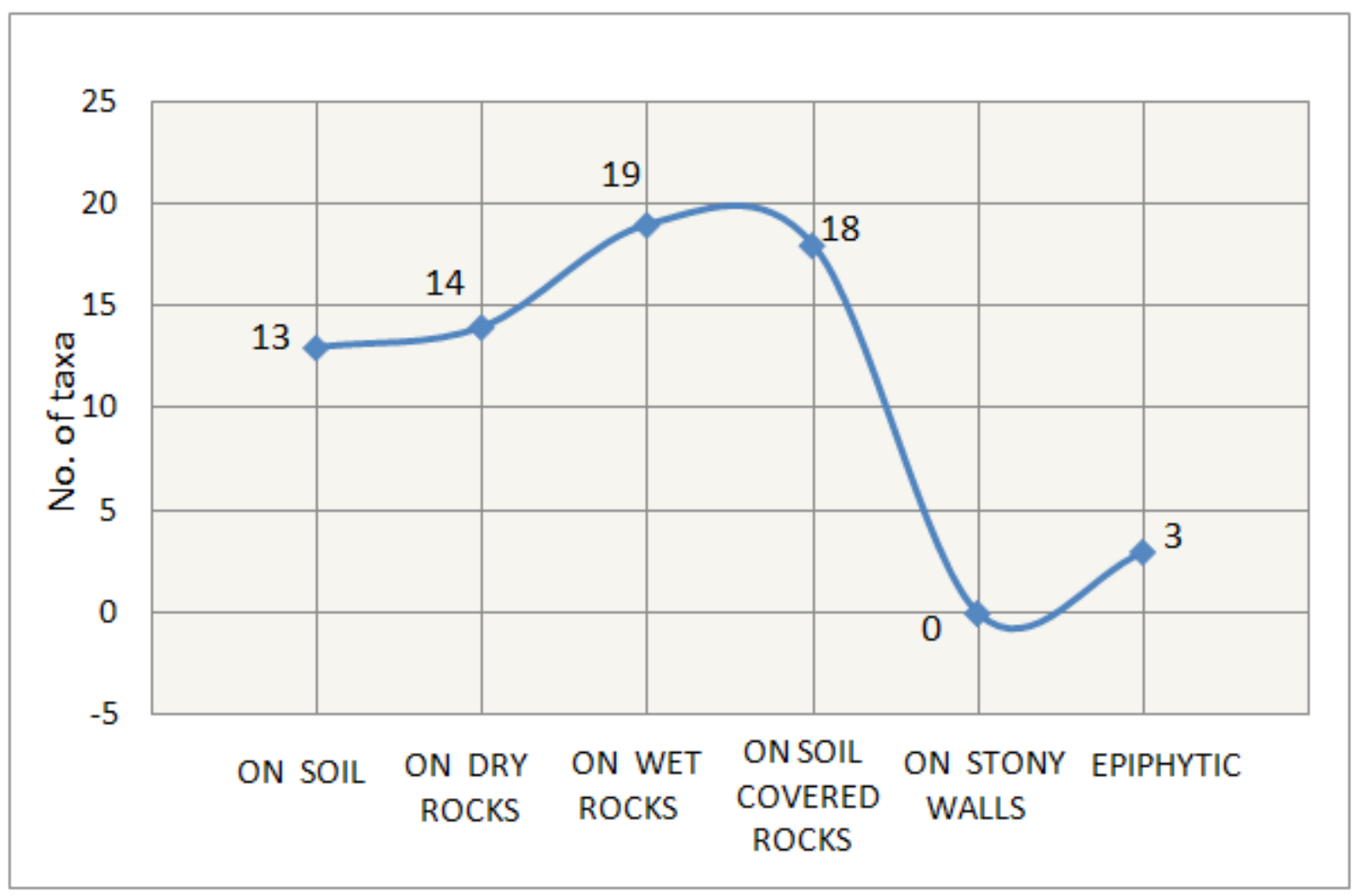

Fig. 1. Habitat wise distribution of liverworts at PBR

studies of bryophyte flora have been done keeping in mind the taxonomic parameters and morphological assessment of the species. The PBR region exhibits variable topography and numerous applicable habitats and the region is at an elevation of 300-1400 $\mathrm{m}$ a.s.l, hence assessment of the distributional pattern of plants of the region is an interesting aspect. The present study deals primarily with this aspect of liverworts of PBR and aims at elucidating the important trends and patterns of liverwort distribution across the altitudinal gradient and at different selected habitats of this reserve area. Taxonomic diversity of liverworts has been discussed emphasizing the dominant families and genera and elaborating the habitat and altitudinal distribution of the taxa. The present work aims to discuss the habitat differentiation of liverworts in PBR and assess the possible effects of the altitudinal gradient on the liverwort distribution.

\section{Materials and Methods}

Plant collections were done in successive years from 30 sites of PBR covering an altitudinal range of approx. 300 to 1400 meters a.s.l. The total study area was divided into three altitudinal zones and from each zone, equal number of sites were selected for collection of bryophytes. The liverwort diversity was assessed on six selected habitats at each site viz. soil, wet rocks, dry rocks, soil covered rocks, stony walls (terricolous habitats) and epiphytic habitat. The collections have been deposited in the Bryophyte Herbarium, National Botanical Research Institute, Lucknow (LWG), India.

\section{Results and Discussions}

During the study, 41 liverworts belonging to 21 genera and 15 families were encountered. In general, rocks, both moist and dry formed the most pertinent habitat for the liverworts, with 33 species being present on rocks. Soil covered rocks also formed preferential habitat followed by soil (Fig. 1). Only three epiphytic taxa were encountered, viz. Lophozia mayebarae (S. Hatt.) N. Kitag., Lejeunea wightii Lindenb. and Frullania ericoides (Nees ex Mart.) Mont. Stony/ brick walls did not harbor any taxa probably due to lack of proper substratum that can bind the thallus.

Among the three altitudinal zones, 17 taxa were found at lower altitudinal gradient $(400-800 \mathrm{~m})$ whereas 12 liverworts were found at the higher altitudinal gradient (1001-1400 m). Thirty three taxa were present at the middle altitudinal zone $(801-1000 \mathrm{~m})$. The sites at middle altitudes furnished amicable conditions for the growth of bryophytes (Fig. 2). Maximum number of species were common in distribution at middle and lower altitudes. Common taxa were also present at middle and higher altitudes. However, less species were common at sites of collection situated at low and high altitudes, indicating the dissimilar species 


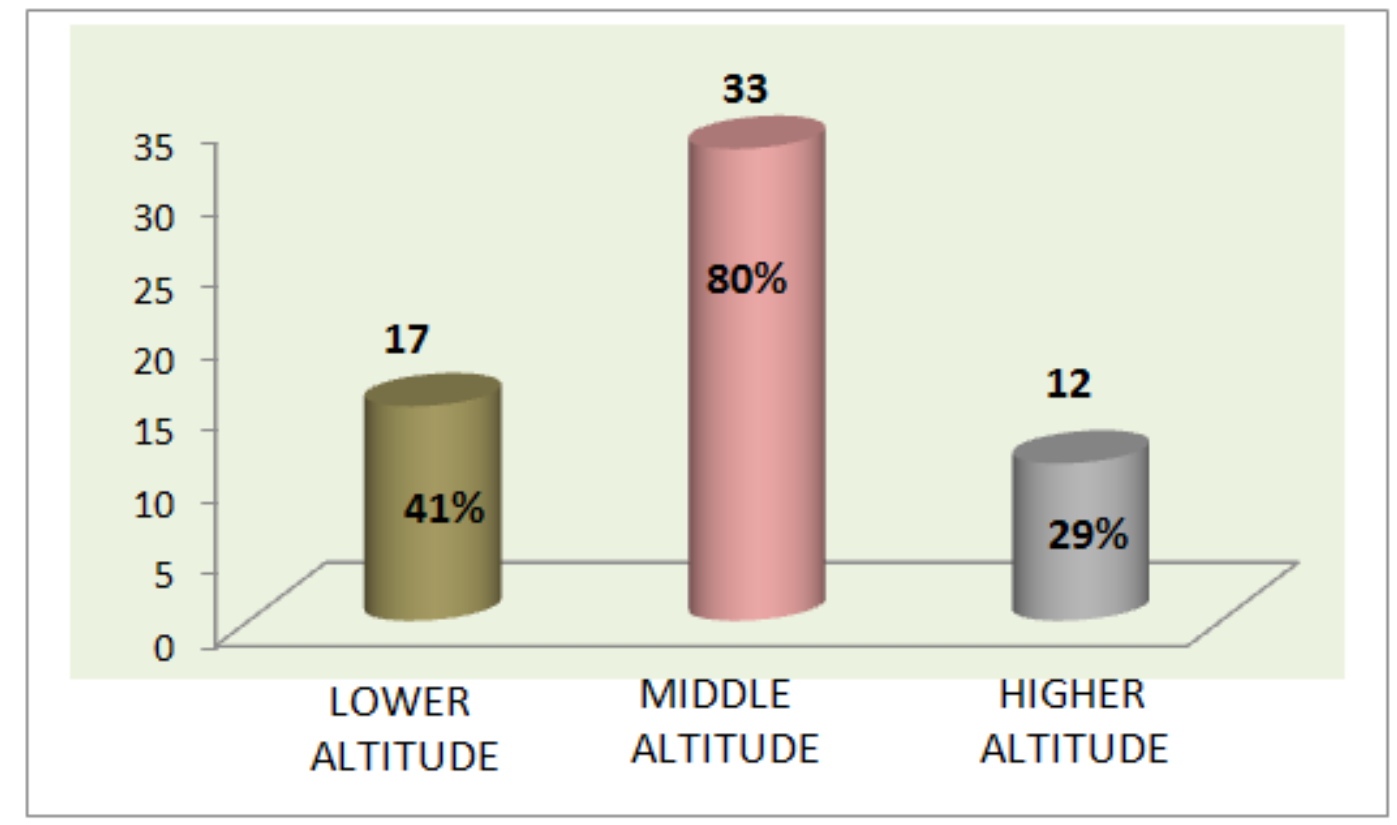

Fig. 2. Species distribution of liverworts across the altitudes at PBR

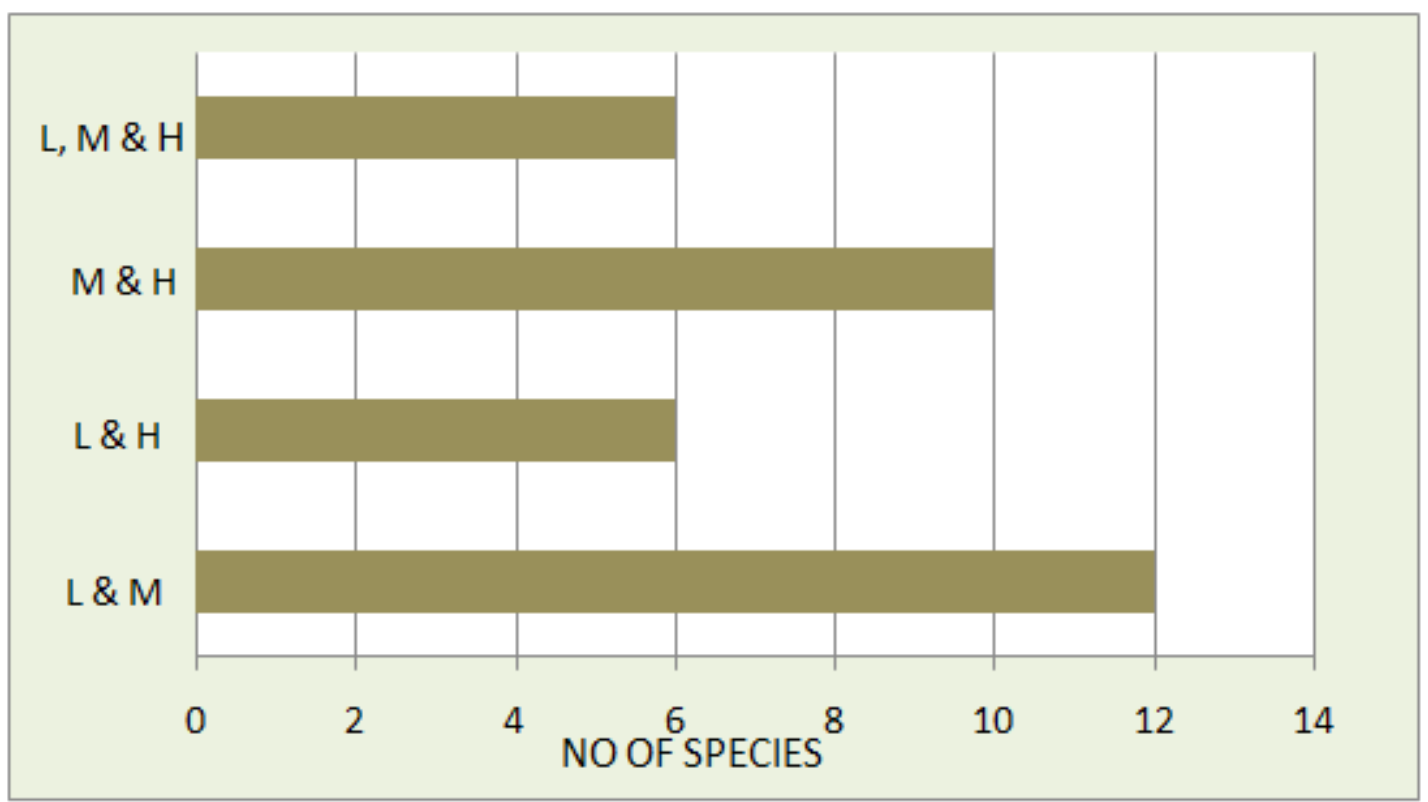

Fig. 3. Liverwort species common to the altitudinal zones at PBR

distribution at low and high altitudes of the area (Fig. 3).

Regarding the liverwort diversity in PBR, six liverwort taxa, viz. Asterella wallichiana (Lehm. \& Lindenb.) Grolle, Dumortiera hirsuta (Sw.) Nees., Marchantia papillata Raddi. subsp. grossibarba (Steph.) Bisch., Pallavicinia lyellii (Hook.) Gray, Plagiochasma appendiculatum Lehm. \& Lindenb. and Targionia hypophylla L. were the most competitive taxa being present at numerous sites, varied habitats and at all the three altitudinal gradients. Among the 15 liverwort families, 11 families (73\%) were represented by a single genus. These were: Fossombroniaceae, Pallaviciniaceae, Aneuraceae, Radulaceae, Marchantiaceae, Conocephalaceae, Targioniaceae, Cyathodiaceae,
Dumortieriaceae, Ricciaceae and Jubulaceae. In terms of species numbers, the dominant families were Jungermanniaceae and Aytoniaceae with 12 and 7 species respectively followed by Aneuraceae (4 species) and Fossombroniaceae (3 species). In case of genera, $66 \%$ were represented by single species viz., Pallavicinia S. Gray, Jungermannia L., Lophozia (Dumort.) Dumort., Chiloscyphus Corda, Heteroscyphus Schiffn., Lejeunea Lib., Lopholejeunea (Spruce) Schiffn., Radula Dumort., Reboulia Raddi, Conocephalum Hill, Targionia L., Cyathodium Kunze, Dumortiera Nees. and Frullania Raddi. The liverwort diversity of Pachmarhi region forms an important part of the cryptogam diversity prevailing at the region. 
Table 1: Observations of the study

HABITATS

S.

No. NAME OF LIVERWORTS

\begin{tabular}{|c|c|c|c|c|c|c|c|c|}
\hline \multirow{2}{*}{$\begin{array}{c}\text { ON } \\
\text { SOIL }\end{array}$} & \multicolumn{2}{|c|}{ ON ROCKS } & \multirow{2}{*}{$\begin{array}{c}\text { ON } \\
\text { SOIL } \\
\text { COVER } \\
\text { ED } \\
\text { ROCKS }\end{array}$} & \multirow{2}{*}{$\begin{array}{c}\text { ON } \\
\text { STON } \\
\text { Y } \\
\text { WAL } \\
\text { L }\end{array}$} & \multirow{2}{*}{$\begin{array}{c}\text { EPIPH- } \\
\text { YTIC/ } \\
\text { ON } \\
\text { DEAD } \\
\text { LOG }\end{array}$} & \multirow{2}{*}{$\begin{array}{c}\text { LOWER } \\
\text { ALTITU } \\
\text { DE } \\
400-800\end{array}$} & \multirow{2}{*}{$\begin{array}{c}\text { MIDDLE } \\
\text { ALTITU } \\
\text { DE } \\
\mathbf{8 0 1 - 1 0 0 0}\end{array}$} & \multirow{2}{*}{$\begin{array}{c}\text { HIGHER } \\
\text { ALTITU } \\
\text { DE } \\
1001- \\
1400\end{array}$} \\
\hline & DRY & $\begin{array}{c}\text { WE } \\
\mathbf{T}\end{array}$ & & & & & & \\
\hline
\end{tabular}

\begin{tabular}{|c|c|c|c|c|c|c|c|c|c|c|}
\hline 1 & $\begin{array}{l}\text { Fossombronia } \\
\text { himalayensis Kashyap }\end{array}$ & - & + & - & + & - & - & + & + & - \\
\hline 2 & $\begin{array}{l}\text { Fossombronia kashyapii } \\
\text { S.C. Srivast. \& Udar }\end{array}$ & + & - & + & - & - & - & - & + & + \\
\hline 3 & $\begin{array}{l}\text { Fossombronia } \\
\text { wondraczekii (Corda) } \\
\text { Dumort. }\end{array}$ & - & - & - & + & - & - & + & - & - \\
\hline 4 & $\begin{array}{l}\text { Pallavicinia lyelli (Hook.) } \\
\text { Gray }\end{array}$ & + & + & + & + & - & - & + & + & + \\
\hline 5 & Riccardia levieri Schiffn. & - & - & + & - & - & - & - & + & - \\
\hline 6 & $\begin{array}{l}\text { Riccardia platyclada } \\
\text { Schiffn. }\end{array}$ & - & - & + & + & - & - & + & + & - \\
\hline 7 & $\begin{array}{l}\text { Riccardia santapaui Udar } \\
\text { \& S.C. Srivast. }\end{array}$ & - & - & + & - & - & - & - & + & - \\
\hline 8 & $\begin{array}{l}\text { Riccardia teneuicostata } \\
\text { Schiffn. }\end{array}$ & - & - & + & - & - & - & - & + & - \\
\hline 9 & $\begin{array}{l}\text { Jungermannia pyriflora } \\
\text { var. major (S. Hatt.) Amak. }\end{array}$ & + & - & + & - & - & - & - & + & - \\
\hline 10 & $\begin{array}{l}\text { Lophozia mayebarae (S. } \\
\text { Hatt.) N. Kitag. }\end{array}$ & - & - & - & - & - & + & - & + & - \\
\hline 11 & $\begin{array}{l}\text { Solenostoma } \\
\text { appressifolium (Mitt.) Váňa } \\
\text { \& D.G. Long }\end{array}$ & - & + & - & - & - & - & - & + & - \\
\hline 12 & $\begin{array}{l}\text { Solenostoma atrobrunnea } \\
\text { (Amak.) Váňa \& D.G. Long }\end{array}$ & - & - & + & - & - & - & + & - & - \\
\hline 13 & $\begin{array}{l}\text { Solenostoma gollanii } \\
\text { Steph. }\end{array}$ & - & - & - & + & - & - & + & - & - \\
\hline 14 & $\begin{array}{l}\text { Solenostoma hyalinum } \\
\text { (Lyell) Mitt. }\end{array}$ & + & + & - & + & - & - & + & + & - \\
\hline 15 & $\begin{array}{l}\text { Solenostoma limbatifolium } \\
\text { (Amak.) Váňa \& D.G. Long }\end{array}$ & - & - & + & - & - & - & - & + & - \\
\hline 16 & $\begin{array}{l}\text { Solenostoma rotundatum } \\
\text { Amak. }\end{array}$ & - & - & + & - & - & - & + & - & - \\
\hline 17 & $\begin{array}{l}\text { Solenostoma } \\
\text { rubripunctatum (S. Hatt.) } \\
\text { R. M. Schust. }\end{array}$ & - & - & - & + & - & - & - & + & - \\
\hline 18 & $\begin{array}{l}\text { Solenostoma subrubrum } \\
\text { (Steph.) Váňa \& D.G. Long }\end{array}$ & - & - & + & - & - & - & - & - & + \\
\hline 19 & $\begin{array}{l}\text { Solenostoma tetragonum } \\
\text { (Lindenb.) R.M. Schust. ex } \\
\text { Váňa \& D.G. Long }\end{array}$ & + & + & + & - & - & - & - & + & + \\
\hline
\end{tabular}




\begin{tabular}{|c|c|c|c|c|c|c|c|c|c|c|}
\hline 20 & $\begin{array}{l}\text { Solenostoma truncatum } \\
\text { (Nees) R.M. Schust. ex } \\
\text { Váňa \& D.G. Long }\end{array}$ & + & - & + & + & - & - & - & + & + \\
\hline 21 & $\begin{array}{l}\text { Chiloscyphus himalayensis } \\
\text { (A. Srivast. \& S.C. Srivast.) } \\
\text { J.J. Engel }\end{array}$ & - & + & - & - & - & - & - & + & - \\
\hline 22 & $\begin{array}{l}\text { Heteroscyphus argutus } \\
\text { (Nees) Schiffn. }\end{array}$ & - & - & + & - & - & - & - & + & - \\
\hline 23 & $\begin{array}{l}\text { Lejeunea wightii } \\
\text { Lindenb. }\end{array}$ & - & - & - & - & - & + & - & + & - \\
\hline 24 & $\begin{array}{l}\text { Lopholejeunea abortiva } \\
\text { (Mitt.) Steph. }\end{array}$ & - & + & - & - & - & - & - & - & - \\
\hline 25 & Radula javanica Gottsche & - & + & - & + & - & - & - & + & - \\
\hline 26 & $\begin{array}{l}\text { Asterella khasiana (Griff.) } \\
\text { Grolle }\end{array}$ & - & - & - & + & - & - & - & + & - \\
\hline 27 & $\begin{array}{l}\text { Asterella leptophylla } \\
\text { (Mont.) Grolle }\end{array}$ & - & - & + & - & - & - & - & - & + \\
\hline 28 & $\begin{array}{l}\text { Asterella multiflora } \\
\text { (Steph.) Pandé, K.P. } \\
\text { Srivast. \& Sultan Khan }\end{array}$ & - & - & - & + & - & - & - & + & - \\
\hline 29 & $\begin{array}{l}\text { Asterella wallichiana } \\
\text { (Lehm.) Grolle }\end{array}$ & - & + & + & + & - & - & + & + & + \\
\hline 30 & $\begin{array}{l}\text { Plagiochasma } \\
\text { appendiculatum Lehm. \& } \\
\text { Lindenb. }\end{array}$ & + & + & + & + & - & - & + & + & + \\
\hline 31 & $\begin{array}{l}\text { Plagiochasma } \\
\text { intermedium Lindenb. \& } \\
\text { Gottsche }\end{array}$ & + & - & - & - & - & - & + & + & - \\
\hline 32 & $\begin{array}{l}\text { Reboulia hemisphaerica } \\
\text { (L.) Raddi }\end{array}$ & - & - & - & + & - & - & - & + & - \\
\hline 33 & $\begin{array}{l}\text { Marchantiapa pillata } \\
\text { subsp.grossibarba (Steph.) } \\
\text { Bischl. }\end{array}$ & + & + & - & + & - & - & + & + & + \\
\hline 34 & $\begin{array}{l}\text { Marchantia paleacea } \\
\text { Bertol. subsp. paleacea }\end{array}$ & - & - & + & + & - & - & + & + & - \\
\hline 35 & $\begin{array}{l}\text { Conocephalum conicum(L.) } \\
\text { Underw. }\end{array}$ & - & + & - & - & - & - & - & + & - \\
\hline 36 & Targionia hypophylla L. & - & + & - & + & - & - & + & + & + \\
\hline 37 & $\begin{array}{l}\text { Cyathodium cavernarum } \\
\text { Kunze }\end{array}$ & + & - & - & + & - & - & + & + & - \\
\hline 38 & $\begin{array}{l}\text { Dumortiera hirsuta (Sw.) } \\
\text { Nees }\end{array}$ & + & - & + & - & - & - & + & + & + \\
\hline 39 & $\begin{array}{l}\text { Riccia billardieri Mont. \& } \\
\text { Nees ex Gottsche, Lindenb. } \\
\& \text { Nees }\end{array}$ & + & - & - & - & - & - & + & - & - \\
\hline 40 & Riccia fluitans L. & + & + & - & - & - & - & - & + & + \\
\hline 41 & $\begin{array}{l}\text { Frullania ericoides (Nees } \\
\text { ex Mart.) Mont. }\end{array}$ & - & - & - & - & - & + & - & + & - \\
\hline
\end{tabular}




\section{Competing Interest}

The authors declare that they have no competing interests.

\section{Authors' contributions}

AKA designed and coordinated the study. RG investigated the specimens, conducted the diversity analysis along the habitats and altitudinal gradients.

\section{Acknowledgements}

The authors are grateful to the Director, National Botanical Research Institute, Lucknow, India for encouragement and providing the laboratory facilities and the Chief Conservator of Forests, Madhya Pradesh, for extending the necessary help during the course of field excursions.

\section{References}

Kaul, A., A. K. Pareek and D. Jain. 1995. Exormotheca tuberifera Kash. A new report from central India. In: S.S. Kumar (ed.) Recent studies on Indian Bryophytes. Bishen Singh Mahendra Pal Singh, Dehra Dun, India. pp. 141-145.

Kaul, A., D. Jain and A. K. Pareek. 1995. Asterella khasiana (Griff.) Grolle. A new report for Pachmarhi. In: S. S. Kumar (ed.) Recent studies on Indian Bryophytes. Bishen Singh Mahendra Pal Singh, Dehra Dun, India. pp. 249-252.
Lal, J. and N. S. Parihar. 1979. Contributions to the Bryoflora of central Indian Zone ILiverworts. J. Indian Bot. Soc. 582): 110114.

Pande, S. K. and K. P. Srivastava. 1952. The hepatic vegetation of Pachmarhi (M.P.): A preliminary survey. Palaeobotanist 1: 368381.

Patidar, K. C., A. Kaul and C. M. Solanki. 1985. Two species of Riccia from Pachmarhi. J. Econ. Taxon. Bot. 6(3): 723-724.

Jain, D. and A. Kaul. 1985. New addition of leafy Jungermanniales in hepatic flora of Pachmarhi. Proceedings of the $73^{\text {rd }}$ Indian Science Congress pp. 69-70.

Kaul, A. 2001. An assessment of the present position of Hepatic flora of Pachmarhi. In: V. Nath \& A. K. Asthana (eds.) Perspectives in Indian Bryology. Bishen Singh Mahendra Pal Singh, Dehra Dun, India. pp.75-85.

Sharma, D. and A. Alam. 2011. Present status of liverwort diversity at Pachmarhi (Madhya Pradesh). J. Indian bot. Soc. 90 (3\&4): 332338.

Singh, V. P. and A. Kaul. 2002. Biodiversity and Vegetation of Pachmarhi Hills. Scientific Publishers, Jodhpur, India. pp. 1-353. 